\title{
MiR-543 Promotes Migration, Invasion and Epithelial-Mesenchymal Transition of Esophageal Cancer Cells by Targeting Phospholipase A2 Group IVA
}

\author{
Huaying Zhao ${ }^{a}$ Changying Diao ${ }^{a} \quad$ Xiaohui Wang ${ }^{a} \quad$ Yilin Xie $^{a}$ Yaqing Liu ${ }^{a}$ \\ Xianzheng $\mathrm{GaO}^{\mathrm{a}}$ Jing Han ${ }^{\mathrm{a}}$ Shenglei $\mathrm{Li}^{\mathrm{a}}$ \\ aDepartment of Pathology, The First Affiliated Hospital of Zhengzhou University, Zhengzhou, Henan, \\ China
}

\section{Key Words}

MiR-543 • PLA2G4A • Esophageal squamous cell carcinoma

\begin{abstract}
Background/Aims: The aim of this study was to investigate the roles of miR-543 and phospholipase A2 group IVA (PLA2G4A) in cell mobility and the invasiveness cascade in esophageal squamous cell carcinoma (ESCC) and to validate the interactive relationship between miR-543 and PLA2G4A. Methods: Microarray analysis showed the different expression levels of PLA2G4A in two ESCC cell lines (KYSE30 and KYSE180). The expression levels of miR543 and PLA2G4A in ESCC tissues were confirmed by qRT-PCR and Western blotting. The targeted relationship between miR-543 and PLA2G4A was studied and verified by a luciferase activity assay. Then, the invasion and metastasis ability of ESCC cell lines transfected with miR543 mimics, miR-543 inhibitor, or PLA2G4A and miR-543 mimics were analyzed separately by Transwell migration and invasion assays. In addition, the roles of miR-543 and PLA2G4A in the expression of E-cadherin and vimentin were also investigated. Results: PLA2G4A upregulated the level of E-cadherin and down-regulated the level of vimentin, which curbed ESCC cell mobility and invasion. In ESCC cells, the expression of miR-543 was significantly higher, whereas the expression of PLA2G4A was markedly lower. MiR-543 facilitated ESCC cell mobility and invasion by repressing PLA2G4A. Conclusions: MiR-543 enhanced the cell mobility and the invasiveness cascade in ESCC cells via the down-regulation of PLA2G4A expression.

(C) 2018 The Author(s)

Published by S. Karger AG, Basel
\end{abstract}

\section{Introduction}

Esophageal squamous cell carcinoma (ESCC) is recognized as a prevalent malignancy with a high morbidity rate [1]. It is the cancer with the sixth highest mortality rate among males and the ninth highest mortality rate among females worldwide. In addition, the

\begin{tabular}{ll}
\hline Shenglei Li & Department of Pathology, The First Affiliated Hospital of Zhengzhou University \\
& No. 1 East Jianshe Road, Erqi District, Zhengzhou, Henan, 450000, (China)
\end{tabular}

Tel. +86 0371-66295532 E-Mail gg11965@126.com 


\section{Cellular Physiology Cell Physiol Biochem 2018;48:1595-1604

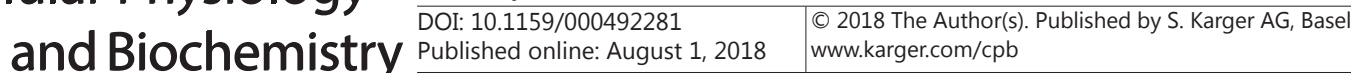 \\ Zhao et al.: Effects of Mir-543/PLA2G4A on ESCC Cells}

total five-year survival rate of ESCC is less than $25 \%$ because of massive invasion into the lymphatic system and distant metastases [2]. The current treatment of ESCC is not effective against invasion and migration. The main reason for the lack of an effective treatment is that the processes of invasion and migration of ESCC cells are complex, with numerous events that are not clearly understood $[3,4]$. Our experiment aimed to deepen the understanding of the process regulating invasion and migration in ESCC.

MicroRNAs (miRNAs) are a class of endogenous and non-coding RNAs [5]. They upregulate or down-regulate their target genes' expression post-transcriptionally by binding to the 3'-untranslated regions (3'-UTRs) of the mRNAs [6]. Several studies have identified by there are many kinds of miRNAs with variable expression in human tumor tissues [7]. As we know from other studies, some miRNAs act as tumor facilitators, whereas others act as inhibitors [8]. Although these two types of miRNAs lead to different results, they both play decisive roles in various aspects of the progression of carcinogenesis, including invasion and migration $[3,9]$. Previous studies on miR-543 showed that it exerts an inhibitive influence in several cancers such as breast cancer and endometrial cancer, while in malignant hepatoma, it acts as a tumorigenesis promoter $[10,11]$. The miR-543 cluster is in the DLK1-DIO3 region on human chromosome 14.

In ESCC, the expression levels of different miRNAs vary. Oncogenic miRNAs, including miR-92a, miR-31 and miR-21, are generally overexpressed in ESCC, while the expression levels of miRNAs that are often considered tumor suppressors, such as miR-145, miR-143 and miR-133a/b, are generally decreased [3]. It has been verified that miR-543 is markedly overexpressed in ESCC. However, the role of miR-543 in the progression of ESCC has not been systematically studied yet.

PLA2G4A is a possible target gene for miR-543. The enzyme that PLA2G4A encodes, as one member of the cytosolic phospholipase A2 group IV family, is related to the regulation of hemodynamics, inflammatory responses and other intracellular pathways. The enzyme catalyzes the hydrolysis of membrane phospholipids to release arachidonic acid [12-14]. Previous studies have already proved that PLA2G4A expression is significantly associated with invasion depth [15], and down-regulation of PLA2G4A might be related to poor patient survival in ESCC. However, the mechanism by which PLA2G4A expression is down-regulated has not yet been studied [16].

This study sought to clarify the biological function of miR-543 in ESCC cell mobility and invasiveness, to identify the exact relationship between miR-543 and PLA2G4A, and to determine the mechanism by which miR-543 and PLA2G4A jointly regulate the progression of ESCC.

\section{Materials and Methods}

Patients

Esophageal squamous cell carcinoma (ESCC) and adjacent normal pancreatic tissue samples $(>5 \mathrm{~cm})$ were obtained from 47 patients hospitalized at the First Affiliated Hospital of Zhengzhou University. None of the patients received treatment before the radical surgical treatment. All sample tissues were frozen in liquid nitrogen and then stored at $-80^{\circ} \mathrm{C}$ until use. Clinicopathologic data (Table 1 ) were collected, including age, sex, degree of differentiation, TNM stage and lymphatic metastasis (positive or negative). All data were obtained from the patients' medical records. The study was approved by the Medical Ethics Committee of the First Affiliated Hospital of Zhengzhou University. Informed consent was obtained from each patient.

\section{Cell cultures}

The ESCC cell lines KYSE30 and ECA-109, and the normal esophageal cell line Het-1A were acquired from BeNa Culture Collection (BNCC, Beijing, China). The ESCC cell lines TE-13 and TE-8 were purchased from Shanghai Enzyme Research Biotechnology Co, Ltd. (Shanghai, China). The ESCC cell line KYSE180 was purchased from KeLei Biological Technology Co, Ltd. (Shanghai, China). HEK-293T cells were purchased from the stem cell bank of the Chinese Academy of Sciences. KYSE30 cells were cultured in $90 \%$ F-12 (Invitrogen) and $10 \%$ FBS (Invitrogen). KYSE180, Het-1A and HEK-293T cells were cultured in $90 \%$ high 


\section{Cellular Physiology Cell Physiol Biochem 2018;48:1595-1604 \begin{tabular}{l|l|l} 
DOI: 10.1159/000492281 & and Biochemistry 2018 The Author(s). Published by S. Karger AG, Basel \\
Published onlne: August 1, 2018 & $\begin{array}{l}\text { www.karger.com/cpb } \\
\text { and }\end{array}$
\end{tabular} \\ Zhao et al.: Effects of Mir-543/PLA2G4A on ESCC Cells}

glucose DMEM (Invitrogen) and $10 \%$ FBS. ECA-109, TE-13 and TE-8 cells were cultured in $90 \%$ RPMI-1640 and $10 \%$ FBS.

\section{Microarray analysis}

The mRNA expression was analyzed by GSE67510 microarray from the GEO public database, where in two pairs of sublines (30-U/D and 180-U/D) with distinct motility capacities were established from two ESCC cell lines (KYSE30 and KYSE180). In addition, aberrantly expressed mRNA was screened by an $\mathrm{R}$ language analysis technique with fold change values more than 2 and $P$ less than 0.05 as the screening conditions.

\section{Cell transfection}

MiR-543 mimics, miR-543 inhibitor, and pcDNA3.1-PLA2G4A were obtained from GenePharma (Shanghai, China). We chose KYSE30 and ECA-109 cell lines for the in vitro transfection experiment. Cells in the logarithmic growth phase were trypsin-digested after resuspension by adding the complete medium prior to transfection. The cells were inoculated into 6-well plates $\left(1 \times 10^{6}\right.$ cells $)$ and incubated for $18-24 \mathrm{~h}$ in an incubator containing $5 \% \mathrm{CO}_{2}$. Cell transfection was carried out with the Lipofectamine 2000 (Life Technologies) system, according to the manufacturer's instructions. Then, all cells were incubated for $48 \mathrm{~h}$ in a $5 \% \mathrm{CO}_{2}$ incubator at $37^{\circ} \mathrm{C}$.

\section{qRT-PCR}

Total RNA isolation was performed by the TRIzol reagent (Invitrogen). The RNA purity and concentration were qualified. The extracted RNA was reverse transcribed using a first strand synthesis kit (Thermo Fisher Scientific) before qRT-PCR was performed. PCR was carried out following the Maxima SYBR Green qPCR Master Mix (2X) kit (Thermo) protocol. Step One Software v2.1 was applied to analyze the results, and the relative gene expression levels were mea-

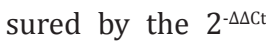
method. The RNA primers used were produced by Sangon Biotech (BBI, Shanghai China) and are displayed in Table 2.
Table 1. The relationship between PLA2G4A expression and clinicopathological characteristics in esophageal squamous cell carcinoma. ${ }^{*} \mathrm{P}<0.05$ was recognized as a significant difference

\begin{tabular}{|c|c|c|c|c|c|}
\hline \multirow{2}{*}{ Item } & \multirow{2}{*}{ Characteristic } & \multirow{2}{*}{ Data $(N=47)$} & \multicolumn{2}{|c|}{ PLA2G4A expression } & \multirow{2}{*}{$P$ value } \\
\hline & & & low & high & \\
\hline \multirow{2}{*}{ Gender } & Male & 36 & 24 & 12 & 0.477 \\
\hline & Female & 11 & 6 & 5 & \\
\hline \multirow{2}{*}{ Age } & $<60$ & 25 & 13 & 12 & 0.547 \\
\hline & $\geq 60$ & 22 & 12 & 10 & \\
\hline \multirow{3}{*}{ Tumor location } & Upper & 4 & 2 & 2 & 0.416 \\
\hline & Middle & 32 & 24 & 8 & \\
\hline & Lower & 11 & 7 & 4 & \\
\hline \multirow{3}{*}{ Tumor grade } & G1 & 2 & 2 & 0 & 0.365 \\
\hline & G2 & 38 & 30 & 8 & \\
\hline & G3 & 7 & 4 & 3 & \\
\hline \multirow{2}{*}{ TNM stage } & I-II & 21 & 12 & 9 & 0.601 \\
\hline & III & 26 & 15 & 11 & \\
\hline \multirow{2}{*}{ Lymphatic metastasis } & Positive & 37 & 31 & 6 & $0.039 *$ \\
\hline & Negative & 10 & 6 & 4 & \\
\hline \multirow{2}{*}{ Distant metastasis } & Yes & 7 & 4 & 3 & $0.048^{*}$ \\
\hline & No & 40 & 35 & 5 & \\
\hline
\end{tabular}

Table 2. Primer sequences for qRT-PCR

\begin{tabular}{lcc}
\hline & Forward $\left(5^{\prime}-3^{\prime}\right)$ & Reverse (5'-3') \\
\hline PLA2G4A & GCTCGGACAGTGATGATGAAT & ACGATGAATCCAACTTGCTTGA \\
miR-543 & ATGAGAAGTTGCCCGTGTT & GCGAATGTTTCGTCACAAAT \\
GAPDH & CGGAGTCAACGGATTTGGTCGTAT & AGCCTTCTCCATGGTGGTGAAGAC \\
U6 & ATTGGAACGATACAGAGAAGATT & GGAACGCTTCACGAATTTG \\
\hline
\end{tabular}




\section{Cellular Physiology Cell Physiol Biochem 2018;48:1595-1604 \begin{tabular}{l|l} 
and Biochemistry Pul: 10.1159/000492281 & $\begin{array}{l}\text { (c) } 2018 \text { The Author(s). Published by S. Karger AG, Basel } \\
\text { www.karger.com/cpb }\end{array}$
\end{tabular} \\ Zhao et al.: Effects of Mir-543/PLA2G4A on ESCC Cells}

Western Blot

Total protein was dissolved by RIPA (radio immunoprecipitation assay) lysate. Then, a BCA protein assay kit was used to calculate the protein concentration. The proteins were subjected to SDS-PAGE and blotted on a PVDF membrane, followed by incubation in the primary antibodies anti-phospholipase A2 (ab58375, 1:500), anti-E-cadherin (ab15148, 1:500), and anti-vimentin (EPR3776, 1:5000) overnight at 4 ${ }^{\circ} \mathrm{C}$. After being washed three times by TBS, the membranes were incubated with the HRP-labeled goat antirabbit IgG (ab6721, Abcam) antibody for $1.5 \mathrm{~h}$. The chromogenic reaction of the membranes was tested by ECL Plus from Life Technology. GAPDH was used as the control.

\section{Nude mice allogeneic experiments}

5-week-old nude mice were purchased from the Guangdong Medical Laboratory Animal Center (Guangdong, China) and were randomly divided into two groups. All mice were injected with KYSE30 cells with either p-PLA2G4A or p-NC. The serum-free cell suspension $\left(1 \times 10^{7} / \mathrm{ml}\right)$ of KYSE30 cells transfected with p-PLA2G4A or p-NC was injected subcutaneously into the back of each mouse $(0.2 \mathrm{~mL})$. When a tumor had grown to approximately $100-200 \mathrm{~mm}^{3}$, the tumor volume was calculated by the following formula: $1 / 2$ $\mathrm{x} \mathrm{L}^{2} \mathrm{x} \mathrm{W}$, where $\mathrm{L}$ is the length $(\mathrm{mm})$, and $\mathrm{W}$ is the width $(\mathrm{mm})$ of the tumor. The average tumor volume and tumor weight were measured every 3 days for a total of 3 times. At the termination of the experiment (the 21st day), the mice were sacrificed, and the tumors were excised from each mouse to measure the average tumor volume and weight. The tumor tissues were used to perform qRT-PCR analysis to determine the expression of PLA2G4A.

\section{Luciferase activity assay}

The possible microRNA target PLA2G4A was identified by TargetScan. The 3'-UTR segment of PLA2G4A was cloned into the plasmid pGL3 at the XbaI and FseI loci. Next, 293T cells were placed in 24-well plates, and transfection was carried out using the plasmid containing the PLA2G4A 3'-UTR wild-type sequence or the mutant sequence. Meanwhile, miR-543 mimics or control mimics were co-transfected by the Lipofectamine 3000 transfection reagent (Life Technology Corporation, Invitrogen ${ }^{\mathrm{TM}}$, USA). The Renilla luciferase vector pRL-SV50 (Promega) was used to equalize the transfection efficiency. The luciferase activity was determined using the dual-luciferase reporter assay system (Promega) $48 \mathrm{~h}$ after transfection.

Transwell invasion assay

Cells were harvested after trypsin digestion, resuspended into serum-free medium and inoculated into the upper chamber of the Transwell system at $5 \times 10^{3}$ per well in $200 \mu \mathrm{L}$ of serum-free medium, and $500 \mu \mathrm{L}$ of DMEM containing $10 \%$ FBS was added to the lower chamber. After a 24-h incubation, the chambers were rinsed twice in PBS, and the cells were fixed using $4 \%$ paraformaldehyde and subsequently stained with 0.1 $\%$ crystal violet. The upper chamber membrane was gently wiped with a cotton swab, and the image was recorded under a microscope.

Transwell migration assay

After being digested by pancreatic enzymes and washed twice in PBS, $5 \times 10^{3}$ cells were cultured in $1 \%$ FBS medium and then placed into the Transwell chamber. After $24 \mathrm{~h}$, the cells were soaked twice in calcium-free PBS, fixed using methyl alcohol and stained with $0.1 \%$ crystal violet. The cells in the upper chamber were gently wiped out with a cotton swab. Finally, we observed the cells under a microscope and took pictures.

\section{Statistical analysis}

All experiments were performed in triplicate and were independently conducted three times. GraphPad Prism 6.0 (Graphpad Software, USA) was used for the statistical analyses. The differences between the two groups were assessed by Student's t-test, while the differences among multiple groups were compared by one-way ANOVA. A p-value $<0.05$ was considered significant.

\section{Results}

Identification of mRNA expression patterns in ESCC cell lines by mRNA chip

By using information from the GPL570 platform, eight samples of the GSE67510 chip were analyzed ( 4 KYSE30 cell lines and 4 KYSE180 cell lines). The mRNA expression levels in 


\section{Cellular Physiology Cell Physiol Biochem 2018;48:1595-1604

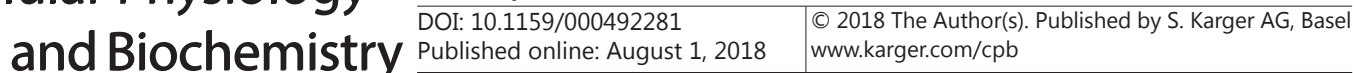 Zhao et al.: Effects of Mir-543/PLA2G4A on ESCC Cells}

the KYSE30 cell lines (Fig. 1A-1B) and the KYSE180 cell lines (Fig. 1C-1D) were screened by the criteria of a fold-change value greater than 2 and $P<0.05$. We identified no intersecting highly expressed genes of the KYSE30 and KYSE180 cell lines (Fig. 1E). We identified two common genes, PLA2G4A and FAM84A, in the KYSE30 and KYSE180 cell lines that had low expression levels (Fig. 1F). PLA2G4A had been researched in other cancers, but it was less studied in esophageal squamous cell carcinoma (ESCC), so we chose PLA2G4A as our research object.

Expression of PLA2G4A in ESCC

The qRT-PCR assay showed that the expression level of PLA2G4A in ESCC tissues was considerably lower compared with the expression level in adjacent normal tissues $(P<0.05$, Fig. 2A). The expression level of the PLA2G4A protein in ESCC tissues was observably lower than that in adjacent normal tissues, as examined by Western blot assay $(P<0.05$, Fig. 2B-C). The relationships between PLA2G4A expression and clinicopathological characteristics in ESCC are shown in Table 1. There was no significant correlation between the expression level of PLA2G4A and clinical features of ESCC patients such as age, gender, and tumor location. However, the expression level of PLA2G4A was significantly correlated with the lymphatic metastasis and distant metastasis of ESCC patients, which indicated that PLA2G4A may affect the

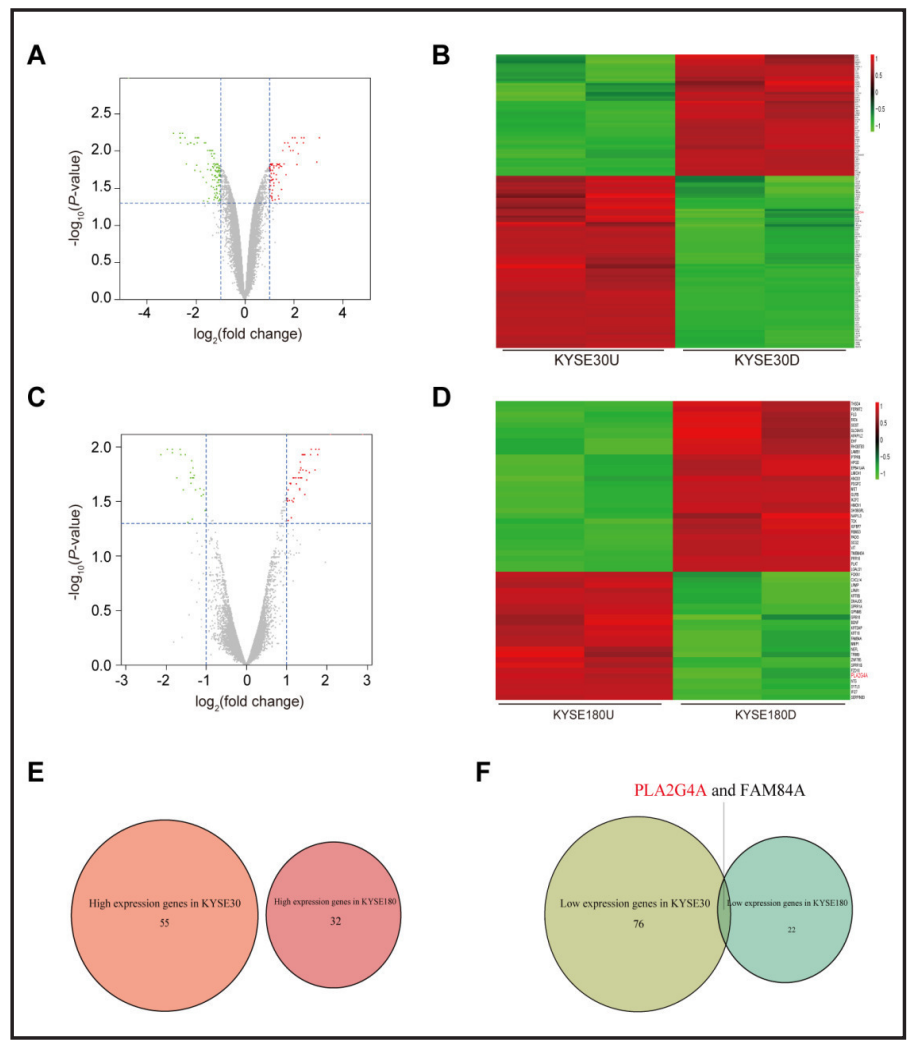

Fig. 1. Identification of mRNA expression patterns in ESCC cell line by mRNA chip(A) Volcano plot of mRNAs in KYSE30. (B) Heatmap of mRNAs screened by fold-change value greater than 2 and $\mathrm{P}<0.05$ in KYSE30. (C) Volcano plot of mRNAs in KYSE180. (D) Heatmap of mRNAs screened by fold-change value greater than 2 and $\mathrm{P}<0.05$ in KYSE180. (E) Venn diagram of highly expressed genes in both KYSE30 and KYSE180. (F) Venn diagram of genes with low expression levels in both KYS30 and KYSE180.
Fig. 2. Low expression of PLA2G4A in ESCC(A) qRT-PCR results of PLA2G4A levels in ESCC tissue and adjacent normal tissue. ${ }^{* *} \mathrm{P}<0.01$, compared with adjacent group. (B-C) Western blot results of PLA2G4A levels in ESCC and adjacent normal tissue. ${ }^{* *} \mathrm{P}<0.01$, compared with adjacent group. (D) Expression level of PLA2G4A mRNA in the ESCC cell lines KYSE30, KYSE180, ECA109, TE-13 and TE-8 and the normal esophageal squamous epithelium Het-1A cell line. ${ }^{*} \mathrm{P}<0.05,{ }^{* *} \mathrm{P}<0.01$, compared with Het-1A. (E) Gray-scale value of protein expression of PLA2G4A in different cell lines. ${ }^{*} \mathrm{P}<0.05,{ }^{* *} \mathrm{P}<0.01$, compared with Het- $1 \mathrm{~A}$.

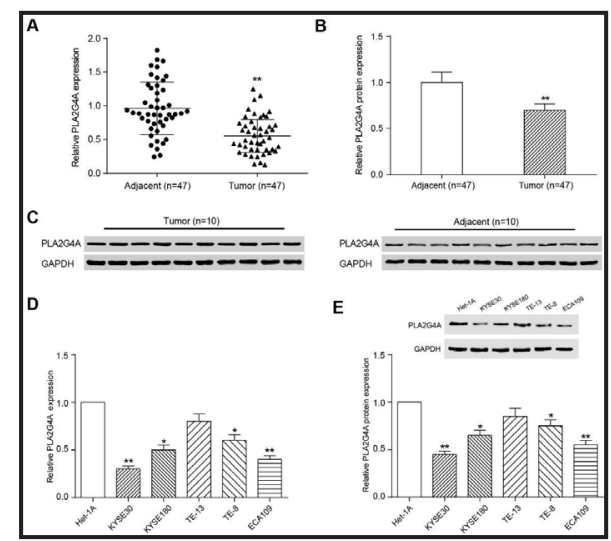


metastasis of ESCC. The mRNA expression levels of PLA2G4A in different ESCC cell lines was analyzed by qRT-PCR. The results showed that the ESCC cell lines KYSE30, KYSE180, ECA109, TE-13 and TE-8 all presented relatively lower expression levels of PLA2G4A mRNA compared with those of normal esophageal squamous epithelium Het-1A cell lines (Fig. 2D). Western blot found that the protein expression of PLA2G4A in ESCC cell lines was also lower than that in normal esophageal squamous cells (Fig. 2E), and the KYSE30 and ECA109 cells lines demonstrated the lowest PLA2G4A protein expression. Therefore, the KYSE30 and ECA109 cell lines, as well as the normal human esophageal squamous epithelium Het-1A cell line were used in the subsequent experiments.

\section{Effect of silencing PLA2G4A on ESCC cell mobility and invasiveness}

The experimental group (p-PLA2G4A group) of KYSE30 and ECA109 cells was transfected with pcDNA3.1-PLA2G4A, and the control group (NC group) of KYSE30 and ECA109 cells was transfected with the empty plasmid. The PLA2G4A expression in KYSE30 and ECA109 cells was significantly increased in the p-HOTAIRM1 group when compared with that in the NC group $(P<0.01$, Fig. 3A-3B). The Transwell results showed that the mobility and invasiveness ability of the KYSE30 and ECA109 cells in the p-PLA2G4A group were notably lower than those of the cells in the NC group $(P<0.01$, Fig. 3C-3D). E-cadherin is a calcium-dependent transmembrane protein that participates in cell-to-cell adhesion and plays a critical part in tumor metastasis. Vimentin is present in mesenchymal cells and is closely related to tumor occurrence and metastasis. Western blot analysis revealed that the protein expression level of E-cadherin in the p-PLA2G4A group was markedly higher than that in the NC group, whereas the protein expression level of vimentin was lower in p-PLA2G4A group than in the NC group $(P<0.01$, Fig. 3E).

PLA2G4A suppressed ESCC growth in vivo

KYSE30 cells transfected with p-PLA2G4A or the empty plasmid were injected subcutaneously into the backs of nude mice. The results of the nude mice allogeneic experiments revealed that the average tumor size in the p-PLA2G4A group was smaller than that of the NC group (Fig. 4A). The average tumor volume was measured every 3 days. The average tumor volume of the p-PLA2G4A group was significantly smaller than that of the NC group (Fig. 4B). At the termination of the experiment (the 21st day), the mice were sacrificed, and the tumors were excised from each mouse to measure the average weight. The average

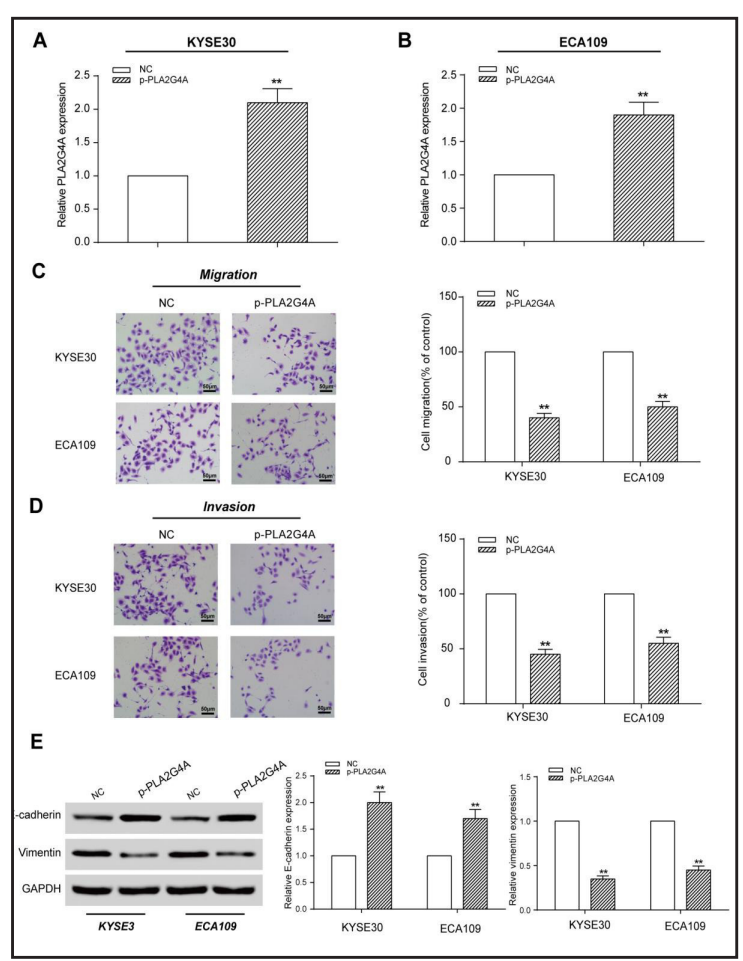

Fig. 3. PLA2G4A reduced invasion and migration of ESCC cells(A-B) PLA2G4A expression levels were detected in KYSE30 (A) and ECA109 (B) cells after transfection with pcDNA3.1-PLA2G4A (p-PLA2G4A) and the empty plasmid (NC) by qRT-PCR analysis. (C) Transwell migration assay results showed that the p-PLA2G4A group down-regulated the migration of KYSE30 and ECA109 cells compared with the NC group. (D) Transwell invasion assay results in KYSE30 and ECA109 cells. (E) Western blot showed that p-PLA2G4A increased the protein expression level of E-cadherin and decreased the protein expression level of vimentin in KYSE30 and ECA109 cells. ${ }^{* *} \mathrm{P}<0.01$, compared with the control group. ${ }^{* *} \mathrm{P}<0.01$, compared with the NC group. 
tumor weight of the p-PLA2G4A group was also significantly less than that of the NC group $(P<0.01$, Fig. $4 C)$. The expression of PLA2G4A was up-regulated approximately $110 \%$ in the p-PLA2G4A group compared with the NC group, as revealed by qRT-PCR $(P<0.01$, Fig. 4D).

\section{MiR-543 was highly expressed in ESCC}

Bioinformatics predicted the binding sites of miR-543 and PLA2G4A (Fig. 5A). In addition, the luciferase activity of cells co-transfected with PLA2G4A wild-type 3'-UTR and miR-543 mimics was decreased in comparison with that of the control group, In contrast, co-transfection with PLA2G4A mutant 3'-UTR and miR-543 mimics had little effect on the luciferase activity $(P<0.01$, Fig. 5A). qRT-PCR analysis demonstrated that the expression level of miR-543 in ESCC tissues was conspicuously higher than in the adjacent normal tissues $(P<0.01$, Fig. 5B). The expression levels of miR-543 in different ESCC cell lines (KYSE30, KYSE180, ECA109, TE-8 and TE-13) were higher than that in the normal esophageal squamous epithelium Het-1A cell line, as revealed by qRT-PCR. In addition, the expression level of miR-543 was the highest in the KYSE30 and ECA109 cells (Fig. 5C). The protein expression level of PLA2G4A in the KYSE30 and ECA109 cells was verified by Western blotting. MiR-543 mimics notably inhibited PLA2G4A protein expression, whereas the miR-543 inhibitor markedly increased PLA2G4A protein expression $(P<0.01$, Fig. 5D).

MiR-543 enhanced the invasion-metastasis cascade of ESCC by inhibiting the expression of PLA2G4A

The expression levels of miR-543 and PLA2G4A were determined in the groups with $\mathrm{NC}$, miR-148a mimics, the miR-148a inhibitor or p-HOTAIRM1 and miR-148a mimics in KYSE30 and ECA109 cells, and the results indicated that the transfection efficiency was satisfactory $(P<0.01$, Fig. 6A-6B).

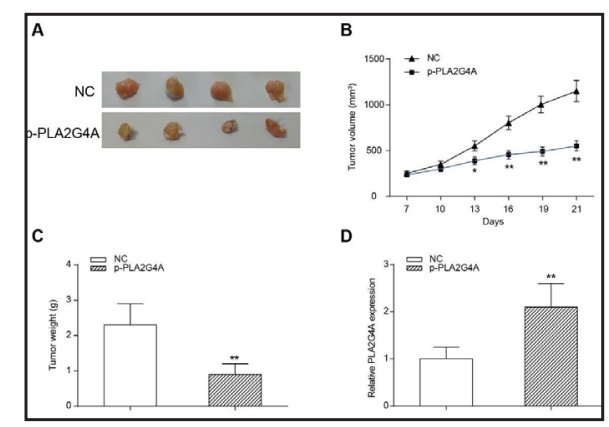

Fig. 4. HOTAIRM1 inhibited tumor formation in nude mice(A) Overexpression of PLA2G4A dramatically suppressed ESCC tumor growth in comparison to that of the NC group. KYSE30 cells were transfected with NC or p-PLA2G4A and inoculated into nude mice, after which the tumor size was monitored. (B) Tumor volumes were measured every 3 days. (C) Tumor weight were measured the 21st day after the injection. (D) The expression level of HOTAIRM1 was significantly higher in ESCC mouse tumor tissue compared with the NC group. ${ }^{* *} \mathrm{P}<0.01$, compared with $\mathrm{NC}$ group.
Fig. 5. MiR-543 was highly expressed in ESCC(A) The binding sites of miR-543 and PLA2G4A were predicted by bioinformatics. Then, luciferase activity analysis indicated that transfection with miR-543 mimics could significantly inhibit the fluorescence activity of the PLA2G4A wild-type luciferase gene. ${ }^{* *}$ $\mathrm{P}<0.01$, compared with the NC group. (B) The qRTPCR results showed high expression levels of miR543 in ESCC tissue. ${ }^{* *} \mathrm{P}<0.01$, compared with the adjacent tissue. (C) Expression levels of miR-543 in ESCC cell lines KYSE30, KYSE180, ECA109, TE-13 and TE- 8 and in the normal esophageal squamous epithelium Het-1A cell line. * $\mathrm{P}<0.05$, ** $\mathrm{P}<0.01$, compared with the Het-1A cell line. (D) Western blot analysis demonstrated the PLA2G4A protein expression levels in KYSE30 and ECA109 cells after transfection with miR-543 mimics and inhibitor. ** $\mathrm{P}<0.01$, compared with the NC group.

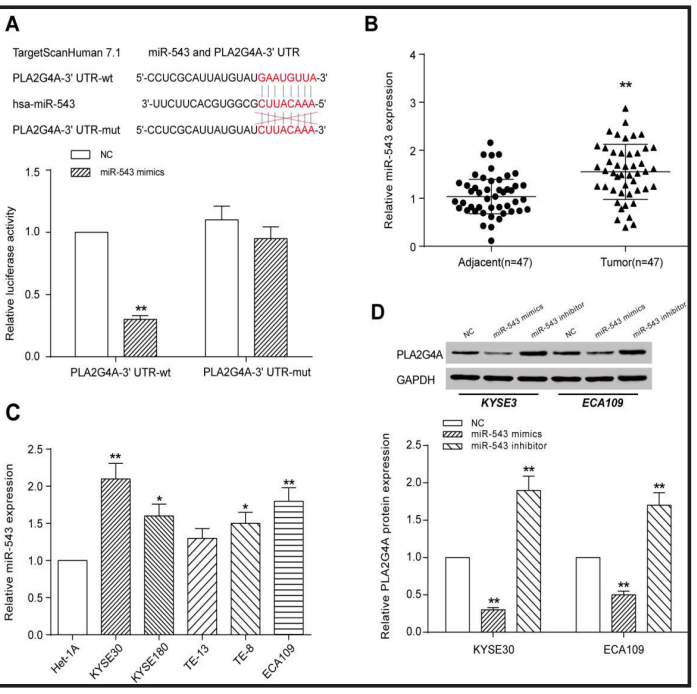




\section{Cellular Physiology Cell Physiol Biochem 2018;48:1595-1604 and Biochemistry Published onl004ne: Aungust 1, $2018 \quad \begin{aligned} & \text { DO 2018 The Author(s). Published by S. Karger AG, Basel } \\ & \text { www.karger.com/cpb }\end{aligned}$ \\ Zhao et al.: Effects of Mir-543/PLA2G4A on ESCC Cells}

The transwell invasion and migration experiments demonstrated that the two cell lines transfected with miR-543 mimics exhibited significantly increased metastasis ability. Moreover, the number of migratory or invasive cells in the miR543 inhibitor group was drastically lower. When PLA2G4A and miR-543 were overexpressed at the same time, the ability of ESCC cells to migrate and invade was returned to the control level $(P<0.01$, Fig. 6C-6D). Western blot indicated that the protein expression level of E-cadherin in the miR-543 mimics group was relatively lower than in the NC group, whereas it was much higher in the miR-543 inhibitor group compared with NC group. The presence of miR-543 mimics in both cell lines could relieve the inhibitory effect of PLA2G4A and allow E-cadherin levels to recover $(P<0.01$, Fig. $6 \mathrm{E})$. The protein expression level of vimentin was higher in the miR-543 mimics group than in the $\mathrm{NC}$ group, while it was lower in the miR543 inhibitor group in comparison with the NC group. Similarly, miR-543 mimics could relieve PLA2G4A inhibition and restore the vimentin levels $(P<0.01$, Fig. $6 \mathrm{~F})$.

\section{Discussion}

Increasing numbers of studies have indicated that miRNAs are involved in various biological processes, such as cell reproduction, differentiation, apoptosis, and metabolism, as well as tumorigenesis [17]. Recently, it has been revealed that altered expression of miRNA contributes to the invasion and metastasis of many cancers $[4,6$, 18]. Increasing numbers of studies have indicated that among numerous other miRNAs, miR-543 is also involved in the processes of initiation and maintenance of many kinds of cancers, including ESCC [17]. It has also been reported that miR543 is down-regulated in breast cancer [19] and endometrial cancer [20] but functions as an oncogene in hepatocellular carcinoma [10,21]. These different conclusions may be caused by differences in the etiology and pathogenesis of these cancers. Increasing numbers of studies have indicated that a poor survival rate in ESCC patients is highly associated with frequent local invasion and distant metastasis [17]. Furthermore, abnormal

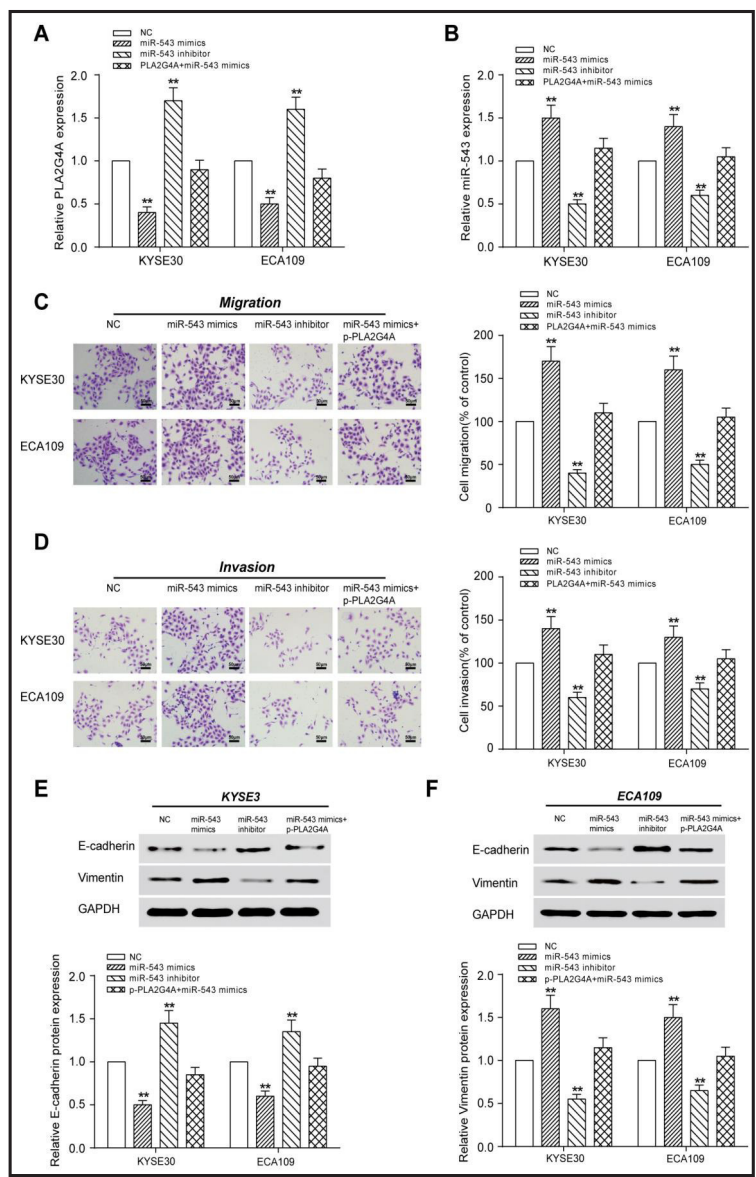

Fig. 6. MiR-543 enhanced invasion-metastasis of ESCC by inhibiting the expression of PLA2G4A(A-B) The expression levels of miR-543 and PLA2G4A in the groups with the NC, miR-148a mimics, miR-148a inhibitor or p-PLA2G4A and miR-148a mimics in KYSE30 and ECA109 cells were determined by qRTPCR. (C) Transwell migration assay results in KYSE30 and ECA109 cells of different transfection groups. (D) Transwell invasion assay results in KYSE30 and ECA109 cells of different transfection groups. (E) Western blot results showed that miR-543 mimics decreased the protein expression levels of E-cadherin, while the miR543 inhibitor increased the protein expression levels of E-cadherin in KYSE30 and ECA109 cells. (F) Western blot results showed that miR-543 mimics increased the protein expression levels of vimentin, while the miR543 inhibitor decreased the protein expression level of vimentin in KYSE30 and ECA109 cells. ** $\mathrm{P}<0.01$, compared with the NC group. 


\section{Cellular Physiology Cell Physiol Biochem 2018;48:1595-1604

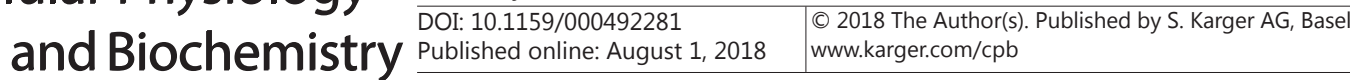

expression of miRNAs has been implicated in the metastasis and progression of cancers by the acquisition of metastatic potential [17]. However, there have been no reports on the expression of miR-543 in ESCC cells or on the role of miR-543 in the invasion and migration of ESCC cells. In this study, the expression level of miR-543 in several ESCC cell lines was determined by qRT-PCR assay, and it was revealed that miR-543 is up-regulated in multiple ESCC cell lines and that it enhances the metastasis of ESCC cells.

Many studies have confirmed that miRNAs can regulate the stability and translation of their target mRNAs by attaching to the 3'UTR of the target [6]. In addition, it has been proven that these miRNAs, including miR-21, miR-138, miR-223 and miR-92a, regulate ESCC development through the regulation of the expression of their target genes, such as PTEN (phosphatase and tensin homolog), FBXW7 (F-box and WD repeat domain containing 7) and PDCD4 (programmed cell death 4) [8]. In this study, we used a luciferase reporter assay to demonstrate that miR-543 was capable of directly targeting the 3'-UTR of PLA2G4A, and the results showed that PLA2G4A is a target of miR-543.

PLA2G4A encodes a calcium-dependent cytosolic phospholipase called cytosolic phospholipase A2 $\alpha$ (cPLA2 $\alpha$ ), which is expressed in a variety of tissues [13]. It is an enzyme that hydrolyzes membrane glycerophospholipids to release arachidonic acid and lysophospholipids that are implicated in cancer cell reproduction [22].. The enzyme that PLA2G4A encodes is also associated with the regulation of hemodynamics, inflammatory responses, and other intracellular pathways [13]. In addition, PLA2G4A is a gene correlated with invasion depth [15]. Some studies have suggested that down-regulation of PLA2G4A expression may be a risk factor for advanced clinic pathological classification and poor patient survival. These findings suggest that it may be possible to use PLA2G4A as an efficient marker for the prediction of outcomes in ESCC patients [16]. Our study showed that PLA2G4A up-regulated the protein expression of E-cadherin and down-regulated the protein expression of vimentin, which inhibited ESCC cell mobility and invasiveness. We also used a Transwell assay to confirm our conclusion.

Nevertheless, there were some limitations of this research. For instance, the specific mechanism by which PLA2G4A regulates the invasion and migration of ESCC cells is worthy of further study, which would help us better understand the process of invasion and migration of ESCC cells. In addition, we conducted our Transwell invasion and migration experiments in KYSE30 and ECA109 cell lines, and experiments in other ESCC cell lines such as KYSE180 and TE-8 should be conducted.

In our study, we confirmed that the expression of PLA2G4A was dramatically inhibited by the overexpression of miR-543, and then we used a Transwell assay to demonstrate that the metastatic ability of ESCC cells was enhanced by miR-543.

\section{Conclusion}

Overall, the present study first confirmed the roles of miR-543 and PLA2G4A in ESCC cell mobility and invasiveness and then demonstrated the targeted relationship between miR-543 and PLA2G4A. The findings of our study could give a better understanding of the mechanism by which the invasion and migration of ESCC cells are regulated by miR-543 and could also indicate a potential target for therapy in the future.

\section{Disclosure Statement}

No conflict of interests exists.

\section{References}

\footnotetext{
1 Zou CD, Zhao WM, Wang XN, Li Q, Huang H, Cheng WP, Jin JF, Zhang H, Wu MJ, Tai S, Zou CX, Gao X: MicroRNA-107: a novel promoter of tumor progression that targets the CPEB3/EGFR axis in human hepatocellular carcinoma. Oncotarget 2016;7:266-278.
} 


\section{Cellular Physiology Cell Physiol Biochem 2018;48:1595-1604 \begin{tabular}{ll|l} 
and Biochemistry Published online: August 1, 2018 & $\begin{array}{l}\text { (c) } 2018 \text { The Author(s). Published by S. Karger AG, Basel } \\
\text { www.karger.com/cpb }\end{array}$
\end{tabular} \\ Zhao et al.: Effects of Mir-543/PLA2G4A on ESCC Cells}

-2 Wang S, Ma G, Zhu H, Lv C, Chu H, Tong N, Wu D, Qiang F, Gong W, Zhao Q, Tao G, Zhou J, Zhang Z, Wang M: miR-107 regulates tumor progression by targeting NF1 in gastric cancer. Sci Rep 2016;6:36531.

3 Jin L, Yi J, Gao Y, Han S, He Z, Chen L, Song H: MiR-630 inhibits invasion and metastasis in esophageal squamous cell carcinoma. Acta Biochim Biophys Sin (Shanghai) 2016;48:810-819.

-4 Zhou YW, Zhang H, Duan CJ, Gao Y, Cheng YD, He D, Li R, Zhang CF: miR-675-5p enhances tumorigenesis and metastasis of esophageal squamous cell carcinoma by targeting REPS2. Oncotarget 2016;7:30730-30747.

-5 Zhou ML, Kang M, Li GC, Guo XM, Zhang Z: Postoperative chemoradiotherapy versus chemotherapy for R0 resected gastric cancer with D2 lymph node dissection: an up-to-date meta-analysis. World J Surg Oncol 2016;14:209.

-6 Wang F, Wang J, Yang X, Chen D, Wang L: MiR-424-5p participates in esophageal squamous cell carcinoma invasion and metastasis via SMAD7 pathway mediated EMT. Diagn Pathol 2016;11:88.

7 Liao J, Liu R, Shi YJ, Yin LH, Pu YP: Exosome-shuttling microRNA-21 promotes cell migration and invasiontargeting PDCD4 in esophageal cancer. Int J Oncol 2016;48:2567-2579.

-8 Shao Y, Li P, Zhu ST, Yue JP, Ji XJ, Ma D, Wang L, Wang YJ, Zong Y, Wu YD, Zhang ST: MiR-26a and miR-144 inhibit proliferation and metastasis of esophageal squamous cell cancer by inhibiting cyclooxygenase- 2 . Oncotarget 2016;7:15173-15186.

9 Ma G, Jing C, Huang F, Li X, Cao X, Liu Z: Integrin alpha6 promotes esophageal cancer metastasis and is targeted by miR-92b. Oncotarget 2017;8:6681-6690.

-10 Fan C, Lin Y, Mao Y, Huang Z, Liu AY, Ma H, Yu D, Maitikabili A, Xiao H, Zhang C, Liu F, Luo Q Ouyang G: MicroRNA-543 suppresses colorectal cancer growth and metastasis by targeting KRAS, MTA1 and HMGA2. Oncotarget 2016;7:21825-21839.

-11 Song N, Liu H, Ma X, Zhang S: Placental growth factor promotes metastases of ovarian cancer through MiR543-regulated MMP7. Cell Physiol Biochem 2015;37:1104-1112.

-12 Gosenca D, Gabriel U, Steidler A, Mayer J, Diem O, Erben P, Fabarius A, Leib-Mosch C, Hofmann WK, Seifarth W: HERV-E-mediated modulation of PLA2G4A transcription in urothelial carcinoma. PLoS One 2012;7:e49341.

13 Hartiala J, Gilliam E, Vikman S, Campos H, Allayee H: Association of PLA2G4A with myocardial infarction is modulated by dietary PUFAs. Am J Clin Nutr 2012;95:959-965.

14 Diouf MN, Sayasith K, Lefebvre R, Silversides DW, Sirois J, Lussier JG: Expression of phospholipase A2 group IVA (PLA2G4A) is upregulated by human chorionic gonadotropin in bovine granulosa cells of ovulatory follicles. Biol Reprod 2006;74:1096-1103.

-15 Zhai YC, Dong B, Wei WQ, He Y, Li XQ Cormier RT, Wang W, Liu F: Overexpression of phospholipase A2 Group IIA in esophageal squamous cell carcinoma and association with cyclooxygenase-2 expression. Asian Pac J Cancer Prev 2014;15:9417-9421.

16 Ren P, Zhang JG, Xiu L, Yu ZT: Clinical significance of phospholipase A2 group IIA (PLA2G2A) expression in primary resected esophageal squamous cell carcinoma. Eur Rev Med Pharmacol Sci 2013;17:752-757.

17 Wang B, Yang J, Xiao B: MicroRNA-20b (miR-20b) Promotes the Proliferation, Migration, Invasion, and Tumorigenicity in Esophageal Cancer Cells via the Regulation of Phosphatase and Tensin Homologue Expression. PLoS One 2016;11:e0164105.

18 Bi M, Chen W, Yu H, Wang J, Ding F, Tang DJ, Tang C: miR-543 is up-regulated in gefitinib-resistant non-small cell lung cancer and promotes cell proliferation and invasion via phosphatase and tensin homolog. Biochem Biophys Res Commun 2016;480:369-374.

19 Haga CL, Phinney DG: MicroRNAs in the imprinted DLK1-DIO3 region repress the epithelial-tomesenchymal transition by targeting the TWIST1 protein signaling network. J Biol Chem 2012;287:4269542707.

20 Bing L, Hong C, Li-Xin S, Wei G: MicroRNA-543 suppresses endometrial cancer oncogenicity via targeting FAK and TWIST1 expression. Arch Gynecol Obstet 2014;290:533-541.

-21 Yu L, Zhou L, Cheng Y, Sun L, Fan J, Liang J, Guo M, Liu N, Zhu L: MicroRNA-543 acts as an oncogene by targeting PAQR3 in hepatocellular carcinoma. Am J Cancer Res 2014;4:897-906.

22 Yao M, Xie C, Kiang MY, Teng Y, Harman D, Tiffen J, Wang Q Sved P, Bao S, Witting P, Holst J, Dong Q: Targeting of cytosolic phospholipase A2alpha impedes cell cycle re-entry of quiescent prostate cancer cells. Oncotarget 2015;6:34458-34474. 